\title{
Gemini 3D infrared spectroscopy: Double nucleus in M 83
}

\author{
Rubén J. Díaz ${ }^{1}$, María P. Agüero ${ }^{1,2}$, Horacio Dottori ${ }^{3}$, \\ Damián Mast ${ }^{1,2}$ and Gustavo Carranza ${ }^{1,4}$ \\ ${ }^{1}$ Observatorio Astronómico de Córdoba, Córdoba, Argentina e-mail: diaz@oac.uncor.edu \\ ${ }^{2}$ SeCyT, Universidad Nacional de Córdoba, Argentina \\ ${ }^{3}$ Instituto de Física, Universidade Federal do Rio Grande do Sul, Porto Alegre, Brazil \\ ${ }^{4}$ CONICET, Argentina
}

\begin{abstract}
We report the status of our study of the central region of M 83. Recently a binary mass concentration at the nucleus was suggested by Thatte et al. (2000), and we determined the possible position of the optically hidden mass concentration with $2 \mathrm{D} \mathrm{H} \alpha$ kinematics. We continue our study of this region trough IR 3D spectroscopy performed with CIRPASS, attached to the Gemini South telescope. Almost 1500 spectra were obtained, centered in the wavelength $1.3 \mu \mathrm{m}$. The velocity field in a region of $13 " \times 9 "$ around the optical nucleus is resolved, with 0.36 " (6.4 pc) sampling. We confirm that the optical nucleus of M 83 is not located at the most important center of symmetry of the velocity field. The largest black hole that could fit to the circular motion in this kinematic center should have a mass less than $2 \times 10^{7} M_{\odot}$.
\end{abstract}

\section{Introduction}

M 83 is a very special case of a grand design barred spiral galaxy that harbours a nuclear X-ray source (Soria \& Wu 2002), coexisting with a massive starburst (e.g. Gallais et al. 1991) in a double circumnuclear ring (Elmegreen et al. 1998) and a secondary mass concentration recently suggested by Thatte et al. (2000). These authors made two long slit observations of the nuclear region using the ISAAC IR spectrometer at the VLT, and detected two peaks in the stellar radial velocity dispersion profile, suggesting the presence of a secondary nucleus. The lack of two-dimensional spectroscopic information did not allow them to fix the precise position of the possible second mass concentration.

The presence of strong kinematical distortions was confirmed by $3 \mathrm{D}$ optical spectroscopic data (Mast et al. 2002). The optical radial velocity and emission lines FWHM fields have distortions that indicate the presence of two mass concentrations, none of them located exactly on the global velocity field minor axis. The mass concentration of the "secondary", hidden nucleus, would be larger than that of the visible one and should be located on the NW (younger and dusty) extreme of the star formation arc, nearby to one of the brightest star forming regions in the galaxy. These previous data compelled us to perform IR 3D spectroscopy, and the preliminary results are presented here.

\section{Gemini IR Observations}

We used the Cambridge Infrared Panoramic Survey Spectrograph (CIRPASS; Parry et al. 2000) operated in the integral field unit (IFU) mode with 490 sampling elements (fibers). The sampling scale in the direct focus $\mathrm{f} / 16$ of the Gemini telescope was 0.36 " $(6.4 \mathrm{pc})$, yielding a sampled field of $13.0 " \times 4.7$ ". The resolution was $R>3000$, in the operating range $0.9-1.8 \mu \mathrm{m}$. 

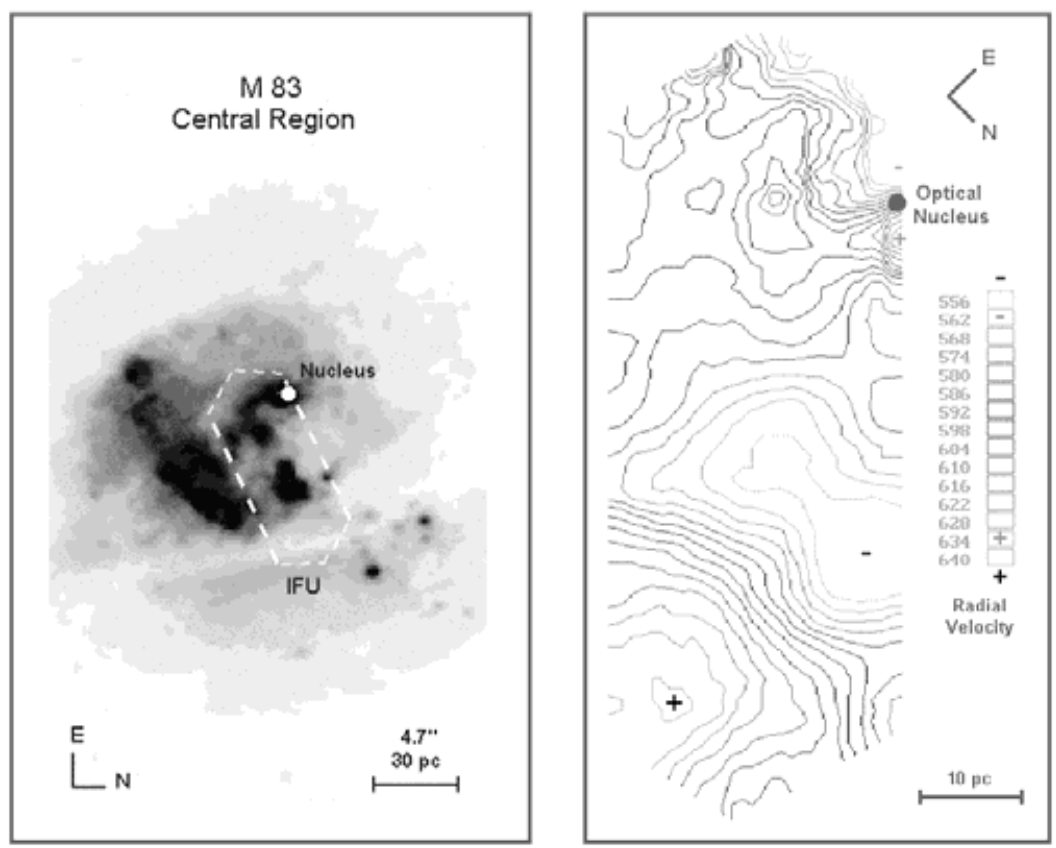

Figure 1. Left: Central region of M 83 (HST, V band), with the first observed IFU position marked with dotted lines. Right: Corresponding $\mathrm{Pa} \beta$ velocity field.

We obtained three integral field spectra of the M 83 core (Figure 1), in the spectral range 1.2-1.4 $\mu \mathrm{m}$, including the emission line $\mathrm{Pa} \beta$. The IFU major axis was oriented at P.A. $120^{\circ}$, and the three fields included the astrometric position of the optical nucleus. The goal was to include both mass concentrations in the total field sampled. The seeing during the observations was about 0.8 ", so the field was slightly oversampled with the 0.36 " fibers set. Details of the reduction process will be published elsewhere. Figure 1 (right) depicts our first $\mathrm{Pa} \beta$ radial velocity field processed (from 490 spectra). The step for the isovelocity lines display was set equal to the average velocity uncertainty. The field corresponds to the zone marked in Figure 1 (left), and a quick comparison of the field with the IFU orientation shows that the largest local rotational feature observed is far from the optical nucleus position, which is in the upper zone of the shown field, and is defined by the continuum emission peak maximum. The largest black hole that could fit to the circular motion in this kinematic center should have a mass less than $2 \times 10^{7} M_{\odot}$. Although different interpretations of these and the previous optical data are possible, the most probable one seems to be that the maximum mass concentration in the core of M 83 is not located at the optical nucleus position.

\section{References}

Elmegreen, D., Chromey, F., \& Warren, A. 1998, AJ, 116, 2834

Gallais, P., Rouan, P., Lacombe, D., Tiphene, D., \& Vauglin, L. 1991, A\&A, 243, 309

Mast, D., Díaz, R., Agüero, M., Weidmann, W., Carranza, G., \& Gimeno, G. 2002, Bol.Asoc.Arg.Ast., 45, 74

Parry, I., et al. 2000, Optical and IR Telescope Instrumentation and Detectors, M. Iye $\mathcal{G} A$. Moorwood Eds., Proc. SPIE, 4008, 1193

Soria, R., \& Wu, K. 2002, A\&A, 384, 99

Thatte, N., Tecza, M., \& Genzel, R. 2000, A\&A, 364, L47 